\section{Probing the character of proteins}

\section{By Michael J. Haas, Senior Writer}

A team from The Scripps Research Institute has developed a high throughput technology that can screen compound libraries against proteins whose biochemical activities are poorly characterized. ${ }^{1}$ The method, which relies on fluorescent activity-based chemical probes to identify hits, can screen libraries containing tens of thousands of compounds, an increase of two orders of magnitude over the capacity of existing activity-based protein binding screens.

About $30-50 \%$ of human proteins have unknown biochemical activity $^{2}$ and thus are not amenable to conventional high throughput screens, which require detailed knowledge of a target protein's activity or binding partners. Activity-based protein profiling (ABPP) overcame these limitations by using chemical probes capable of binding the active site on a large number of mechanistically related enzymes, thus obviating the need for specific information about a given target. But the approach required gel-based assays-a laborious and time-consuming process that limited activity-based screens to small libraries.

The Scripps team set out to overcome this low throughput hurdle by combining two screening approaches: activity-based probes and a microtiter plate-based high throughput screening technique. They dubbed the technique fluorescence polarization technology for competitive ABPP (fluopol-ABPP).

The team dispensed purified target protein into a 384-well micro titer plate, spiked each well with an individual library compound and added a polarized fluorescent probe. This setup allowed the fluorescence signals to be detected in the plate, bypassing the need for gelbased separation and detection.

Using fluopol-ABPP, the team screened libraries against two uncharacterized proteins implicated in human cancers: retinoblastoma binding protein 9 (RBBP9) and glutathione $S$-transferase $\omega 1$ (GSTO1). A screen of a 19,000-member library against RBBP9 identified a lead, the alkaloid emetine, that inhibited the protein at low micromolar concentrations. A screen of a 2,000-member library against GSTO1 identified an $\alpha$-chloroacetamide that inhibited the protein at nanomolar concentrations. Gel-based assays confirmed the results of both screens.

Next, the team screened emetine and 75 structurally similar compounds against RBBP9 with activity-based probes and gel-based detection assays. They used the results to develop structure-activity relationships that shed light on the binding behavior of RBBP9 and the mechanism by which it is inhibited.

"We hope to use the inhibitors discovered by fluopol-ABPP as pharma cological tools to map the biochemical activities of enzymes," team leader Benjamin Cravatt told SciBX. Cravatt is chairman of chemical physiology at Scripps.

The team's report on fluopol-ABPP technology was published in Nature Biotechnology.

\section{Out of the shadows}

Experts contacted by SciBX said the Scripps team's method provided a good starting point for characterizing poorly understood proteins that have roles in human disease. Indeed, the key limitation may lie not in the method itself but in the availability of suitable probes.

Matthew Bogyo, associate professor of pathology at Stanford University School of Medicine, said fluopol-ABPP offered the dual advantages of increasing throughput relative to gel-based assays and simplifying the assay design relative to conventional high throughput approaches.

Bogyo has used activity-based probes to screen libraries containing at most a few thousand compounds and then used a gel-based assay to resolve and identify the hits. "This was certainly a low throughput method-not one I'd want to use on a library" containing tens of thousands of compounds as the Scripps team did in its study, he said.

Bogyo did note that the detection of fluorescence signals in the microtiter plates required screening of purified protein. That involves an information trade-off, he said, because "no offtarget effects or selectivity could be assessed this way." In contrast, he said gel-based assays can screen proteins in mixtures and even in cells, thereby providing some selectivity information about the hits.

The two screening methods don't have to be mutually exclusive, Bogyo added. Fluopol-ABPP "could be a starting point for early-stage drug discovery research. You could use it to get at a scaffold that could lead to basic medicinal chemistry, X-ray crystallography studies and assessment of the biochemical functions of the protein," he told SciBX.

Jinyan Du, a research fellow at Dana-Farber Cancer Institute and the Broad Institute of MIT and Harvard, said fluopol-ABPP "will enable the identification of inhibitors for a much broader range of enzymes," in contrast to existing high throughput screening methods, which focus primarily on well-studied proteins such as kinases.

She said she would be interested in using this technology in her own research because it bypassed the need to identify suitable substrates for the target or develop robust in vitro assays around the target-substrate binding interaction. Du was lead author on a paper published in January that described a high throughput method for profiling activated tyrosine kinases in cancer cells. ${ }^{3}$

According to Du, another benefit of fluopol-ABPP is that use of 
activity-based probes requires no specialized knowledge, only knowledge of the general mechanistic classes of enzymes. "The method uses standard assays and equipment, so its adaptation by medicinal chemists should be quite straightforward," she said.

Bogyo agreed that the technology should be readily exportable to any academic and industrial facility with high throughput screening technology. "This is a great application that has real value," he said. "It will continue to have even more value as more probes become available."

Indeed, Bogyo suggested that the chief limitation of the new technology might be the lack of probes suitable for screening some proteins. Most available activity-based probes target serine or cysteine proteases because these proteins are well known, although he said several researchers are working on probes for different classes of enzymes.

At the same time, Bogyo acknowledged that it might not be necessary to develop new probes to screen new classes of proteins with the Scripps method. "Some existing probes might work well enough on a purified protein target" to yield hits, he said.

Cravatt said that suitable probes are typically straightforward to synthesize and can be produced at sufficient scale and in a cost-effective manner for use with his team's method.
Cravatt said the team is using fluopol-ABPP to screen a range of uncharacterized proteins that appear to play roles in human disease. "We are also interested in expanding the technology to address enzymes from distinct mechanistic classes," he said.

Cravatt added that the team plans to optimize leads from their screens via conventional medicinal chemistry. He declined to disclose the IP status of the findings.

Haas, M.J. SciBX 2(15); doi:10.1038/scibx.2009.604

Published online April 16, 2009

\section{REFERENCES}

1. Bachovchin, D. et al. Nat. Biotechnol.; published online March 29, 2009; doi:10.1038/nbt.1531

Contact: Benjamin F. Cravatt, The Scripps Research Institute, La Jolla, Calif. e-mail: cravatt@scripps.edu

2. Galperin, M. \& Koonin, E. Nucleic Acids Res. 32, 5452-5463 (2004)

3. Du, J. et al. Nat. Biotechnol. 27, 77-83 (2009)

COMPANIES AND INSTITUTIONS MENTIONED

Broad Institute of MIT and Harvard, Cambridge, Mass.

Dana-Farber Cancer Institute, Boston, Mass.

The Scripps Research Institute, La Jolla, Calif.

Stanford University School of Medicine, Stanford, Calif. 